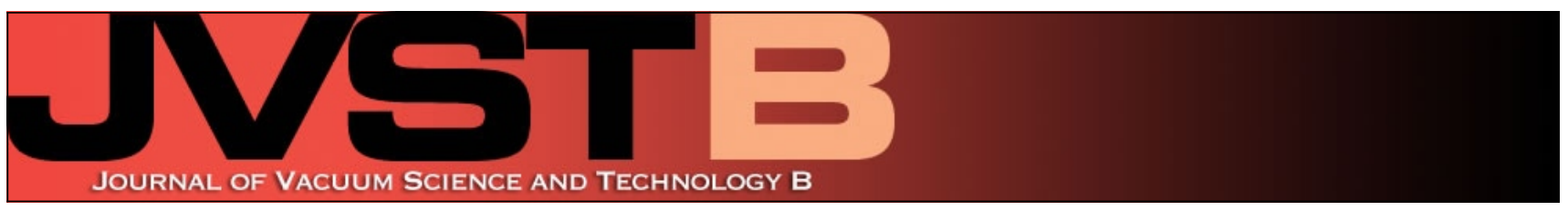

\title{
Independent parallel lithography using the atomic force microscope
}

S. C. Minne, S. R. Manalis, A. Atalar, and C. F. Quate

Citation: J. Vac. Sci. Technol. B 14, 2456 (1996); doi: 10.1116/1.588753

View online: http://dx.doi.org/10.1116/1.588753

View Table of Contents: http://avspublications.org/resource/1/JVTBD9/v14/i4

Published by the AVS: Science \& Technology of Materials, Interfaces, and Processing

\section{Related Articles}

Three dimensional reciprocal space measurement by $\mathrm{x}$-ray diffraction using linear and area detectors:

Applications to texture and defects determination in oriented thin films and nanoprecipitates

J. Vac. Sci. Technol. A 31, 021505 (2013)

The Si3N4/TiN Interface: 3. Si3N4/TiN(001) Grown with a -150 V Substrate Bias and Analyzed In situ using

Angle-resolved X-ray Photoelectron Spectroscopy

Surf. Sci. Spectra 19, 52 (2012)

Facile fabrication of scalable patterned nickel nanocone arrays for field emission applications

J. Vac. Sci. Technol. B 31, 02B104 (2013)

Process considerations for layer-by-layer 3D patterning of silicon, using ion implantation, silicon deposition, and selective silicon etching

J. Vac. Sci. Technol. B 30, 06FF05 (2012)

Tilted nanostructure fabrication by electron beam lithography

J. Vac. Sci. Technol. B 30, 06F302 (2012)

\section{Additional information on J. Vac. Sci. Technol. B}

Journal Homepage: http://avspublications.org/jvstb

Journal Information: http://avspublications.org/jvstb/about/about_the_journal

Top downloads: http://avspublications.org/jvstb/top_20_most_downloaded

Information for Authors: http://avspublications.org/jvstb/authors/information_for_contributors

\section{ADVERTISEMENT}

\section{Instruments for advanced science}
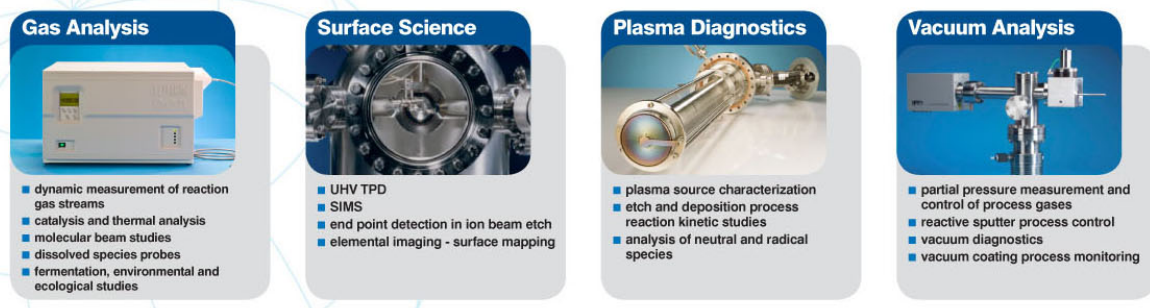

contact Hiden Analytical for further details

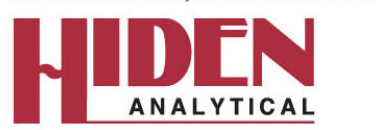

info@hideninc.com

www.HidenAnalytical.com

CLICK to view our product catalogue 


\title{
Independent parallel lithography using the atomic force microscope
}

\author{
S. C. Minne, ${ }^{\text {a) }}$ S. R. Manalis, A. Atalar, ${ }^{\text {b) }}$ and C. F. Quate ${ }^{\text {c) }}$ \\ E. L. Ginzton Laboratory, Stanford University, Stanford, California 94305
}

(Received 15 March 1996; accepted 13 May 1996)

\begin{abstract}
Independent parallel features have been lithographically patterned with a $2 \times 1$ array of individually controlled cantilevers using an atomic force microscope. Control of the individual cantilevers was achieved with an integrated piezoelectric actuator in feedback with a piezoresistive sensor. Patterns were formed on $\langle 100\rangle$ single crystal silicon by using a computer controlled tip voltage to locally enhance the oxidation of the silicon. Using the piezoresistor directly as a force sensor, parallel images can be simultaneously acquired in the constant force mode. A discussion of electrostatic forces due to applied tip voltages, hysteresis characteristics of the actuator, and the cantilever system is also presented. (c) 1996 American Vacuum Society.
\end{abstract}

\section{INTRODUCTION}

The scanning probe microscope (SPM) has demonstrated itself to be a versatile and effective tool for patterning surfaces at the nanometer scale. Two common methods for modifying surfaces using probe microscopes are direct physical patterning and electric field assisted patterning. While both methods of surface modification are quite different, they both require that a sharp tip interacts with the surface to be patterned.

Physical patterning consists of scribing or indenting a sample using the tip of the SPM. Jung ${ }^{1}$ has used this process to scribe patterns into polymer surfaces, and $\mathrm{Mamin}^{2}$ has used the physical indentation process in conjunction with laser heating to store $100 \mathrm{~nm}$ bits at $100 \mathrm{kHz}$ in a polymer surface. This approach has the advantage that the sample is typically much softer and generally unreactive with the tip, thereby reducing tip wear. The literature on electrical modification of surfaces with probe microscopes is much more extensive. Scanning probe lithography was pioneered by Dagata, ${ }^{3}$ who patterned $\langle 111\rangle$ silicon with the scanning tunneling microscope (STM), and Lyding ${ }^{4}$ has used this same technique in ultrahigh vacuum (UHV) to pattern features less than a few nanometers. Snow and Campbell have modified this technique and patterned $\mathrm{Si}^{5}$ and $\mathrm{GaAs}^{6}$ with the atomic force microscope.

This form of lithography can be used to pattern a variety of surfaces. ${ }^{7}$ Using the atomic force microscope (AFM) or STM, various materials have been patterned, including chromium by Song, ${ }^{8}$ titanium by Sugimura, ${ }^{9}$ and amorphous silicon by Kramer. ${ }^{10}$ Recently, we have demonstrated the use of amorphous silicon as a general resist for lithography. ${ }^{11}$ Also, organic materials have been modified by electrical means, as demonstrated by Majumdar $^{12}$ using PMMA. Finally, Sugimura ${ }^{13}$ has used the STM to pattern amorphous silicon and then globally modify the surface by gold electroplating.

Further work in this area has yielded features more complex than simple lines on substrates. Previously we demon-

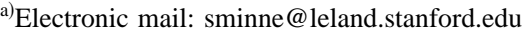

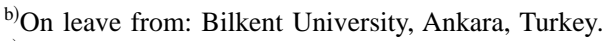

${ }^{c}$ Electronic mail: quate@ee.stanford.edu
}

strated the compatibility of scanning probe lithography with semiconductor processing by fabricating a $0.1 \mu \mathrm{m} n$-metaloxide-semiconductor field-effect transistor $\left(n\right.$ MOSFET $\left.^{14}\right)$ with the AFM. Campbell ${ }^{15}$ has fabricated a side-gated transistor on a silicon-on-insulator wafer, and Matsumoto ${ }^{16}$ has fabricated a single electron transistor by modifying titanium. More recently, Snow ${ }^{17}$ has demonstrated real time lithographic monitoring using AFM lithography in order to fabricate metal-oxide-metal junctions.

While scanning probe lithography has revealed its potential value by fabricating operational devices, it suffers the drawback of being very slow since the scanning probe is an inherently serial device. For this form of lithography to be accepted beyond the single device level, the throughput of this approach must be greatly improved. We have addressed this problem in two ways, by increasing the tip velocity and by devising a parallel scheme.

In general, the tip velocity of scanning probes is governed by the speed of the feedback control of the microscope, which is itself limited by the resonant frequency of the actuator (typically less than $1 \mathrm{kHz}$ ). By integrating the actuator onto the cantilever, it is now possible to work up to near the cantilever resonant frequency $(20-100 \mathrm{kHz})$, allowing the tip velocity to be significantly increased. With our integrated actuator, we have shown tip velocities in feedback operation of up to $3 \mathrm{~mm} / \mathrm{s}$ with a piezoresistive sensor ${ }^{18}$ and up to 1 $\mathrm{cm} / \mathrm{s}$ with optical lever detection. ${ }^{19}$

Increased tip velocity reduces the time that the tip will take to cover a given area, but, even with the increased speed, the time necessary for a single tip to cover a wafer area is unacceptably long. Parallelism is an alternative approach to circumvent this problem. Previously we have used parallel arrays of five cantilevers for imaging and lithography, ${ }^{11}$ but in that work, we did not have individual control of the cantilevers and therefore could not operate each cantilever in feedback. We have since fabricated an array of cantilevers with integrated sensors and actuators and used them for parallel imaging in feedback. ${ }^{20}$ In this article, we advance our previous work on the $2 \times 1$ arrays by modifying their design to show parallel imaging without the need for force correction. We also demonstrate that the areas 


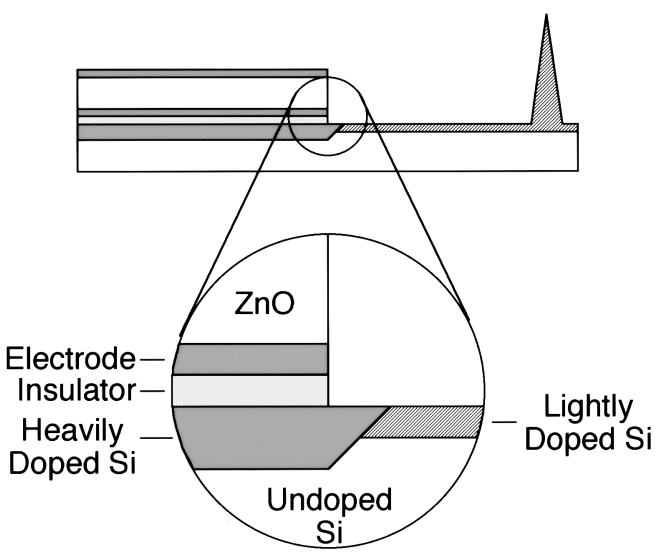

FIG. 1. Schematic cross-sectional view of a piezoresistive cantilever with integrated piezoelectric actuator. The piezoresistor lies on the surface of the silicon, separated from the $\mathrm{ZnO}$ actuator by silicon dioxide and silicon nitride. The piezoresistor doping extends to the apex of the tip.

scanned by the two cantilevers can be simultaneously patterned using a system with feedback and compatible with high speed AFM.

\section{CANTILEVERS}

The cantilevers used in this work are silicon piezoresistive cantilevers with integrated single crystal silicon tips developed by Tortonese. ${ }^{21}$ We have modified the cantilever structure to include a zinc oxide ( $\mathrm{ZnO})$ actuator, which is fabricated on top of the silicon cantilever (Fig. 1). Since $\mathrm{ZnO}$ is piezoelectric, an applied electric field will cause the $\mathrm{ZnO}$ film to expand in the direction of the electric field and contract in the lateral direction. Due to the bimorph nature of the films, this contraction results in bending.

When imaging, a feedback loop monitors the cantilever deflection with the piezoresistor to determine the voltage that the $\mathrm{ZnO}$ actuator needs to maintain constant force between the tip and the sample. The fabrication process and initial results using this type of AFM cantilever with an integrated sensor and actuator are presented in Ref. 20.

The main disadvantage of the system described in Ref. 20 is that the sensor and actuator were not completely mechanically decoupled. Since the piezoresistive sensor extended over the entire length of the cantilever, the portion of the sensor beneath the $\mathrm{ZnO}$ deflects with the $\mathrm{ZnO}$, generating a signal unrelated to the force on the tip. In order to take a feedback image, it is necessary to electronically compensate this mechanical coupling which limited the useful range of the actuator.

In this work, we have modified the cantilever by doping the area beneath the $\mathrm{ZnO}^{22}$ to eliminate sensor-actuator coupling and the need for the correction circuit. A schematic diagram of the side view of the cantilever including the new implant is shown in Fig. 1. The increased doping serves two purposes. The piezoresistive effect ${ }^{23}$ can be described by the following equation:
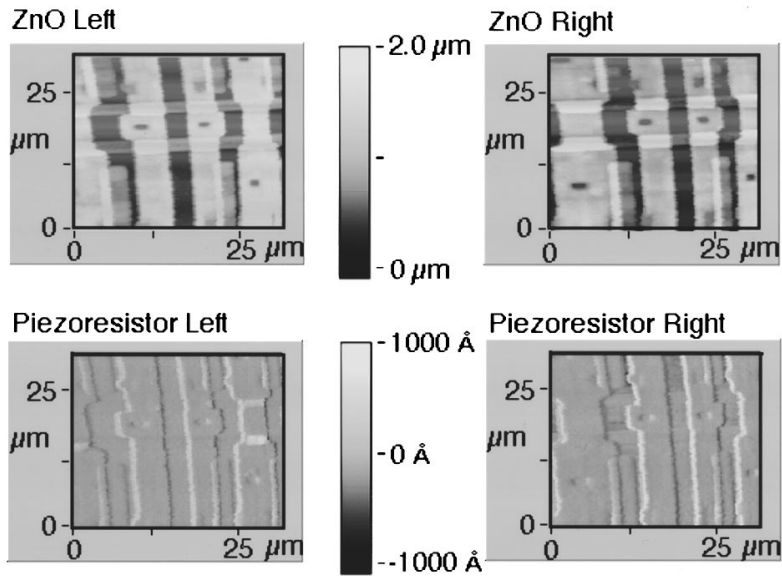

FIG. 2. Parallel constant force AFM image of a microcircuit. The $\mathrm{ZnO}$ maintains constant force over $2 \mu \mathrm{m}$ of topography. Beneath the images ( $\mathrm{ZnO}$ signal) are the corresponding error signals. The reduced error signal scale indicates constant force is being maintained.

$$
\frac{\Delta \rho}{\rho}(x, y, z)=\pi_{L} S(x, y, z),
$$

where $\rho$ is the resistivity of the cantilever material, $\pi_{L}$ is the longitudinal piezoresistive coefficient, and $S$ is the stress. Assuming a constant piezoresistor width and depth, $\Delta R / R$ is found by integrating and normalizing Eq. (1) over the length of the resistor. Our sensing circuitry measures changes in resistance of the entire cantilever (both the area under the $\mathrm{ZnO}$ and the tip area, which contains the active piezoresistive sensor). By increasing the doping under the $\mathrm{ZnO}$ region, we decrease the piezoresistive coefficient $\left(\pi_{L}\right)$ by $80 \%$, a reduction that directly affects the contribution to the sensor signal induced by the actuator. Another improvement comes from the nature of the fractional change. As the doping increases, the resistance decreases, and as a result, the absolute change in resistance from a given stress is reduced.

The small coupling permits imaging without the need for correction circuitry. An uncorrected parallel constant force image of an integrated circuit containing vertical topography of $2 \mu \mathrm{m}$ is presented in Fig. 2. The top images are the voltages controlling the $\mathrm{ZnO}$ actuator and represent the surface topography, while the lower images are the piezoresistor signals which represent the error signals. For constant force imaging the error signals should be zero. The reduced scale on the error signal images shows that constant force is effectively maintained.

The frequency response of the cantilevers has been presented in detail in previous work. ${ }^{18-20}$ The hysteresis and linearity measurements of the cantilever are presented in Fig. 3 . Linearity and hysteresis measurements of the actuator were obtained by measuring the cantilever deflection with an optical level system. The optical system is calibrated to the out of contact movement of the cantilever in the following manner: The tip is brought close to the surface of a calibrated piezotube. The voltage to the $\mathrm{ZnO}$ is ramped until the tip to strikes the sample. The piezotube is then retracted from the tip by a known amount. The amount of additional voltage to 
(a)

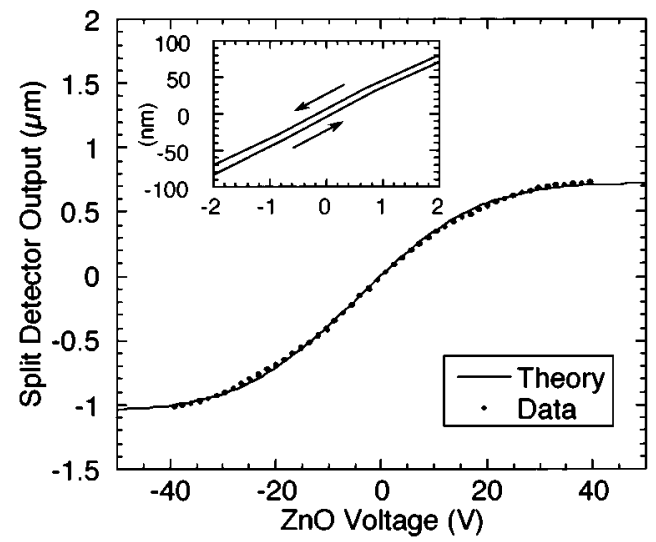

(b)

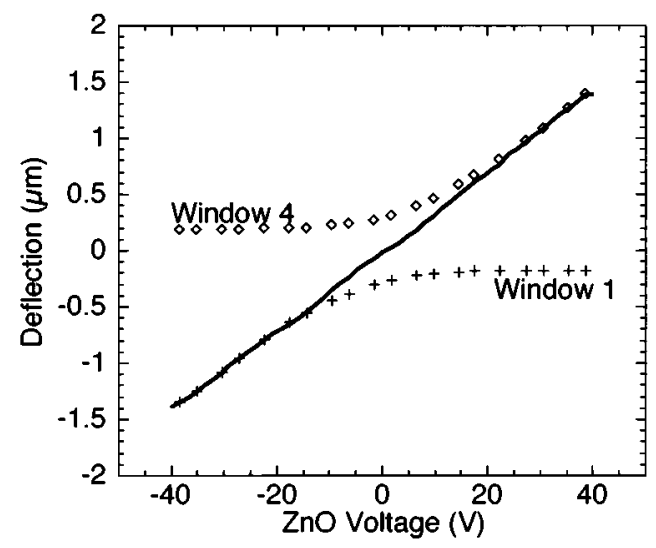

FIG. 3. (a) Hysteresis curve for the $\mathrm{ZnO}$ actuator. The points represent the data, while the solid line is a simulation of a Gaussian beam passing over a split photodiode. Inset: Expanded view of the data around zero applied voltage showing $20 \mathrm{~nm}$ maximum hysteresis. (b) Linearity plot for the $\mathrm{ZnO}$ actuator. The solid line is a composite curve of four photodiode positions representing the overall linearity curve of the actuator. The points of the first and final windows used to construct the overall plot are included. This method was required because the cantilever displaced the beam beyond the linear range of the photodiode.

the $\mathrm{ZnO}$ required to bring the tip back into contact with the surface provides a calibration for tip movement in air for a change in $\mathrm{ZnO}$ voltage. The cantilever is then deflected by the $\mathrm{ZnO}$ in air while the output of the laser is monitored. The slope of the response combined with the previous result gives the change in laser output for a given displacement of a freely suspended tip.

Figure 3(a) shows the output of a split photodetector when the $\mathrm{ZnO}$ voltage is swept from -40 to $40 \mathrm{~V}$ and then back to $-40 \mathrm{~V}$. The inset of Fig. 3(a) shows an expanded view of the hysteresis loop around zero volts applied voltage. The maximum hysteresis of the $\mathrm{ZnO}$ in this voltage range is $20 \mathrm{~nm}$. Unfortunately, it is not possible to determine the linearity of the actuator from Fig. 3(a). The split photodiode detector used to measure the position of the beam reflected off the cantilever has a limited range for which the output is linear with respect to cantilever deflection. This limitation occurs because the size of the reflected beam is finite and must cover an adequate portion of both sides of the detector. In our microscope, the extent of the linear range covers roughly $1 \mu \mathrm{m}$ of deflection and is insufficient to characterize the entire range of the $\mathrm{ZnO}$ actuator. It is interesting to note that the sigmoidal shape of Fig. 3(a) reflects the Gaussian distribution of our laser diode. We have also simulated a Gaussian beam traversing a split photodiode and plotted the results with the data in Fig. 3(a).

In order to measure linearity of the actuator, we manually translated the linear window of the detector in discrete steps so that it covered the entire range of the reflected laser beam. This provided us with a series of curves, each of which has only a small linear range. We then extracted and shifted the linear data from each curve to construct the overall linearity curve. The data was shifted such that overlapping voltages in adjacent windows had the same deflection.

Two such windows, which represent the initial and final data curves used for constructing the overall plot, along with the final construction, are plotted in Fig. 3(b). In these windows, the defector position was adjusted so that response is linear at the maximum excursion of the cantilever. Once the beam is completely deflected to one side of the photodiode, the detector cannot provide information about the cantilever deflection and the curves flatten. The composite curve of Fig. 3(b) shows excellent linearity of the device over its entire range of $3 \mu \mathrm{m}$.

\section{ELECTROSTATIC FORCES}

Electrical AFM lithography involves applying a voltage between the tip and the substrate to be modified. The force produced by the applied voltage is the negative spatial derivative of the energy, $U=\frac{1}{2} C V^{2}$, where $C$ is the capacitance and $V$ is the voltage. The capacitance is primarily a function of the distance between the tip and the sample, and the effective area of the tip.

When the AFM operates in a repulsive mode (contact imaging), the electrostatic attraction produced by an applied voltage between the tip and sample increases the contact force but does not cause the cantilever to deflect. If the sample is soft, the applied voltage provides a method for physical modification using an electronic signal. If the sample is hard, the increased forces accelerate the wear on the delicate tip. Since the detector is sensitive only to cantilever deflection, it is difficult to accurately monitor this attractive force while the tip remains in constant contact with the surface.

In order to measure the contact force produced by the electrostatic attraction, we retracted and extended the sample while monitoring the cantilever deflection with the piezoresistor. A force curve was obtained by plotting the deflection versus vertical position. Figure 4(a) shows a typical force curve where the sample is biased to $-7.5 \mathrm{~V}$ and being retracted from the tip. An additional bias is provided by the piezoresistor measurement process. For this data, the extra bias is $0.6 \mathrm{~V}$ at the tip. The electrostatic attraction can be determined by multiplying the cantilever spring constant by the distance for which the tip is electrostatically fixed to the sample during retraction. The electrostatic force is labeled " $E F$ " on Fig. 4(a). In our experiments, we find that the elec- 
(a)

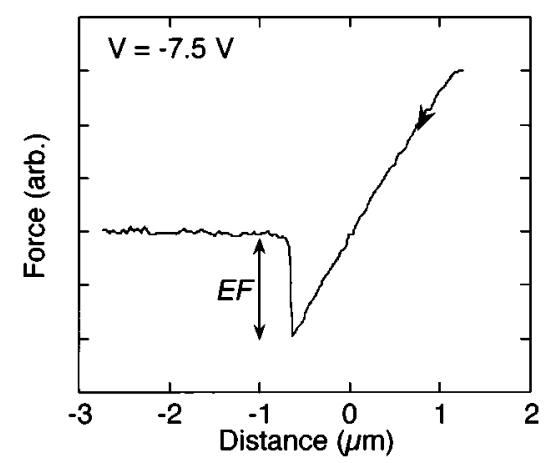

(b)

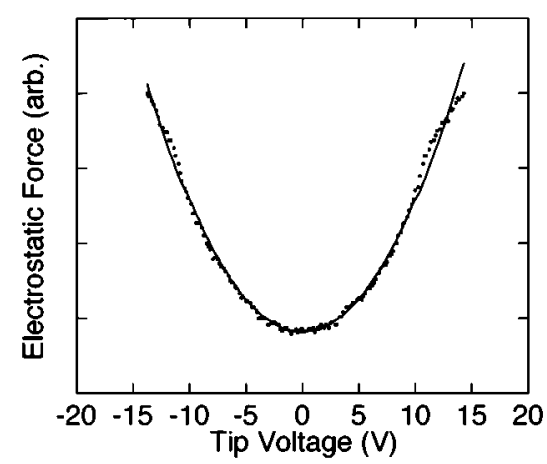

FIG. 4. (a) Force curve trace as the cantilever is retracted from the sample. The sticking is due to the electrostatic attraction of the applied voltage between the tip and sample. (b) The electrostatic attraction between the tip and the sample as a function of applied voltage.

trostatic force is significantly greater than the meniscus force. The electrostatic force can be measured as a function of applied voltage by reproducing the data in Fig. 4(a) for many voltages and is shown in Fig. 4(b). A second order polynomial describes the data well in Fig. 4(b), indicating that the force is proportional to the square of the applied voltage.

Figure 5(a) shows a scanning electron micrograph of the tip used to obtain the data in Fig. 4. This tip initially had a radius of curvature of $\sim 300 \AA$, but due to the process we used to measure the contact force versus voltage, the tip sharpness was reduced to $\sim 1.5 \mu \mathrm{m}$, as shown in Fig. 5(d). During initial measurements made with this tip, we measured a contact force of $20 \mathrm{nN}$ for a voltage of $5 \mathrm{~V}$. As the measurement process was repeated, this force continued to increase until stabilizing at a force of $1.25 \mu \mathrm{N}$. Presumably the tip had dulled to a point that no further damage was occurring. The results of Fig. 4 and 5 indicate that the contact force between the tip and sample under an applied voltage is dependent on the magnitude of the voltage and the degree of tip wear.

Preliminary results show that tip wear can be a significant problem. In our lithographic experiments, we worked with single crystal silicon tips patterning single crystal silicon substrates. Due to the hardness of the substrate and the chemical interaction between silicon tip and silicon sample, and because of the increased force due to the applied voltage,

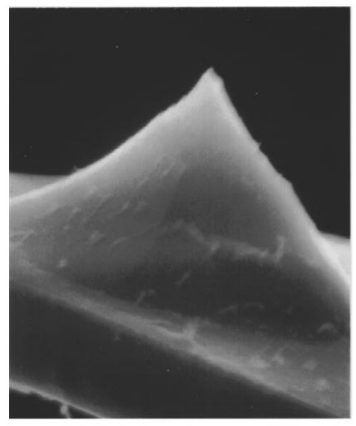

(a)

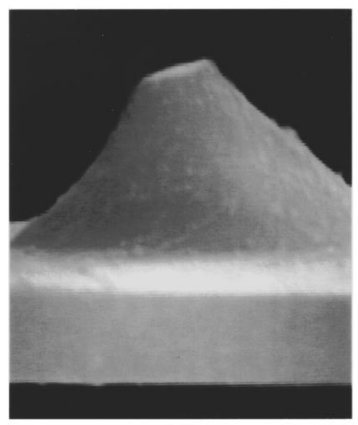

(c)

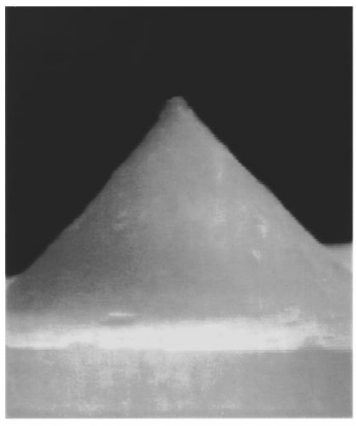

(b)

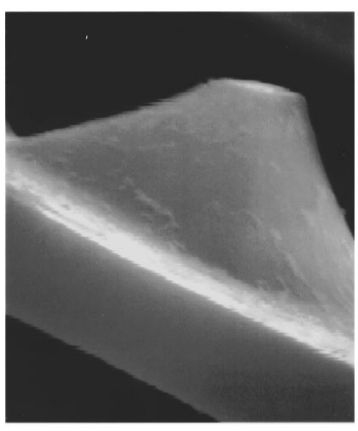

(d)
FIG. 5. SEM micrographs of (a) a new tip, (b) a slightly worn tip which is still useful for silicon lithography, (c) a heavily worn tip which is no longer useful for silicon lithography, and (d) the tip used for the measurement of Fig. 4. In (d), the heavy damage is due to the measurement process described in Fig. 4.

the impact on the tip is substantial. Tips showing various states of wear are shown in Fig. 5. In Fig. 5(a), we show a new tip which has a radius of curvature of $\sim 300 \AA$. Figure 5(b) shows a tip that has performed and is still capable of performing high quality lithography, although the radius of curvature has increased to $\sim 2000 \AA$ A. Figure 5(c) shows a tip that was originally effective in performing lithography but has been worn down to the point where lithography is no longer possible. The surface diameter of the tip is about 1 $\mu \mathrm{m}$. It can be seen that the tip wear is due to the scanning process and is exacerbated by the electrostatic forces from the voltage used for lithography - the straight angle on the worn tip corresponds to the scanning angle of our AFM. We believe that lithography fails as the tip wears because of the decrease in electric field due to the dulling geometry of the tip and because most of the conductive implant in the tip region has worn off.

\section{LITHOGRAPHY}

Our preferred method for performing lithography is to use the conductive path in the piezoresistor to apply appropriate voltages to the conducting tip. Initially the $\mathrm{ZnO}$ was used to control the lithography by biasing the sample and pulling the tip off the surface when lithography was not desired, but this method showed limited success due to the large electrostatic forces. In other attempts, we tried to use the $\mathrm{ZnO}$ as both the sensor and actuator, ${ }^{24}$ and dedicated the piezoresistor for 
lithographic voltages only. While this method provided good imaging capabilities, the lithographic signal would often interfere with the feedback loop. We believe that this approach is still attractive, but at this time our cantilevers are not optimized for the use of the $\mathrm{ZnO}$ as a sensor.

In order to use the piezoresistor as both a sensor and an electrical conductor for lithography, it is necessary to separate the two signals. By using an ac bridge, we can sense piezoresistor deflections at high frequency (typically 100 $\mathrm{kHz}$ ) while sending the electrical pulses for lithography through the cantilever at lower frequencies (filtered to 100 $\mathrm{Hz}$ ). A benefit of this system is that very high scan speeds can be obtained using the ac bridge (see Ref. 18). Another consideration when using the piezoresistor as a path for lithography is electrical breakdown between the piezoresistor and the bottom $\mathrm{ZnO}$ electrode. Referring back to Fig. 1, the piezoresistor is separated from the bottom electrode by a thin film of dielectric. Since the bottom electrode is held at ground, a lithography signal can cause breakdown between the films. It is therefore necessary to bias both $\mathrm{ZnO}$ electrodes and the piezoresistor with the lithography voltage used for writing the lithographic patterns.

Also, for high resolution imaging it is necessary to use a high gain low noise amplifier for sensing the cantilever deflection. Unfortunately, the large voltage needed for lithography normally precludes the use of such amplifiers in our circuitry.

To address the problems of tip speed, dielectric breakdown, and low noise amplification, two transformers and a battery powered amplifier were used in order to decouple the cantilever from ground. By biasing the entire cantilever (both $\mathrm{ZnO}$ electrodes and the piezoresistor) to the lithography voltage, the piezoresistor could be measured at ac without being influenced by the high voltage lithographic signals. Also, by biasing the entire cantilever, breakdown between the films is no longer a concern. Duplicating the electronics for each of the cantilevers within the array, parallel independent lithography in feedback is readily accomplished.

In general, we have found that more reliable pattern generation is obtained by using a combination of open and closed loop feedback. The open loop portion occurs only when the lithography pulse is on. When writing, the $\mathrm{ZnO}$ exerts a force to partially oppose the electrostatic forces in order to reduce tip wear and increase tip lifetime. In this way, cantilevers are maintained in constant force mode as they profile the surface. Only when writing voltage is applied do they pull back from the surface to compensate for the electrostatic force. Once the lithography signal is turned off, the tips return to the normal feedback mode. Lithography has been accomplished using both this mode as well as regular closed loop feedback.

Figure 6(a) shows an optical image of a large scale independent parallel lithographic pattern using the open and closed feedback system. The substrate is $\langle 100\rangle$ silicon and is patterned with a lithographic voltage of $20 \mathrm{~V}$. The presumed mechanism for the lithography is that the large electric field desorbes hydrogen and increases the oxidation rate on the

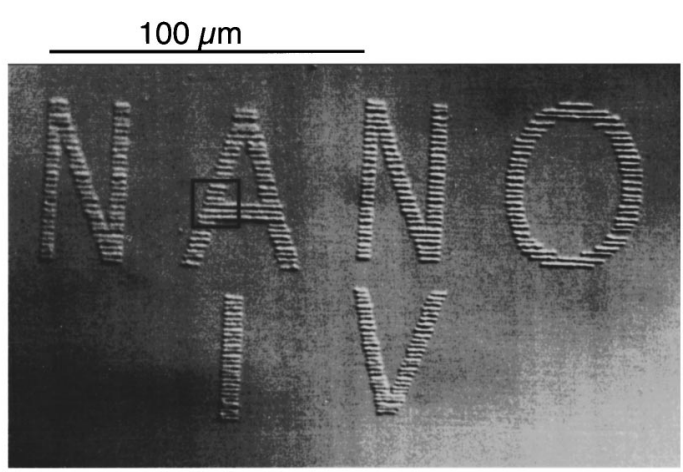

(b)

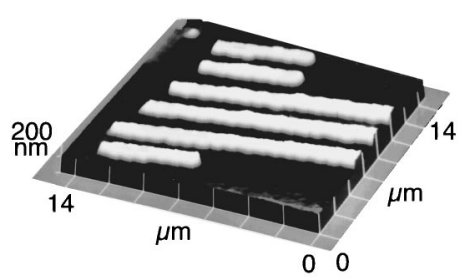

FIG. 6. (a) Independent parallel lithography using cantilevers with integrated sensors and actuators. The lithography covers $200 \mu \mathrm{m} \times 100 \mu \mathrm{m}$ with one tip writing " $\mathrm{N} A$ " over " $\mathrm{I}$ " and the other simultaneously writing "N O" over " $\mathrm{V}$ " in a single pass. (b) Magnified AFM image of (a).

exposed silicon. Details of this process are described elsewhere. ${ }^{3,5}$ The latent image is transferred to the substrate with a $\mathrm{KOH}$ etch. In Fig. 6(a), each tip has traversed a 100 $\mu \mathrm{m} \times 100 \mu \mathrm{m}$ area of the $200 \mu \mathrm{m} \times 100 \mu \mathrm{m}$ total area in a raster pattern. A computer program controlled the scanning and coordinated the lithography voltages. With one pass, the left tip patterned the letters "N A" over the "I" and the right tip patterned the letters "N O" over the "V". Figure 6(b) is an enlarged AFM image of the pattern in the square on Fig. 6(a). The modulation in the lines is caused by the digitization from computer control.

\section{CONCLUSIONS}

Expansion of scanning probe microscope lithography through parallelism, combined with the high scan speeds enabled by integrated actuators, are important steps that begin to address the issue of throughput for a probe based lithography system. Using this approach, we have achieved the first demonstration of parallel, independent AFM lithography using cantilevers with independent control spanning an area of $20000 \mu \mathrm{m}^{2}$.

We believe further improvement in cantilever design can be implemented. Optimization of the $\mathrm{ZnO}$ geometry to improve its simultaneous sensing and actuating capabilities would allow the elimination of the piezoresistor leaving the silicon exclusively for lithographic signal path. Alternatively, adding a separate conducting path to the tip would serve the same function, while allowing us to maintain the current design.

\section{ACKNOWLEDGMENTS}

The authors would like to thank Babur Hadimioglu and Jim Zesch at Xerox PARC for the ZnO deposition and 
Charles Ahn and Nick Ulman for insightful discussions. Primary support for this work came from the Joint Services Electronics Program N0014-91-J-1050 of the Office of Naval Research with partial support from the NSF and CMR at Stanford. S. C. M. acknowledges the support of the Leland T. Edwards Fellowship and S. R. M. acknowledges the support of the Urbanek Fellowship.

${ }^{1}$ T. A. Jung, A. Moser, H. J. Hug, D. Brodbeck, R. Hofer, H. R. Hidber, and U. D. Schwarz, Ultramicroscopy 42, 1446 (1992).

${ }^{2}$ H. J. Mamin and D. Rugar, Appl. Phys. Lett. 61, 1003 (1992).

${ }^{3}$ J. A. Dagata, J. Schneir, H. H. Harary, C. J. Evans, M. T. Postek, and J. Bennett, Appl. Phys. Lett. 56, 2001 (1990).

${ }^{4}$ J. W. Lyding, T. C. Chen, J. S. Hubacek, J. R. Tucker, and G. C. Abeln, Appl. Phys. Lett. 64, 2010 (1994).

${ }^{5}$ E. S. Snow and P. M. Campbell, Appl. Phys. Lett. 64, 1932 (1994).

${ }^{6}$ E. S. Snow, P. M. Campbell, and B. V. Shanabrook, Appl. Phys. Lett. 63, 3488 (1993).

${ }^{7}$ C. R. K. Marrian, Technology of Proximal Probe Lithography (SPIE Optical Engineering, Bellingham, WA, 1993).

${ }^{8}$ H. J. Song, M. J. Rack, K. Abugharbieh, S. Y. Lee, V. Khan, D. K. Ferry, and D. R. Allee, J. Vac. Sci. Technol. B 12, 3720 (1994).

${ }^{9}$ H. Sugimura, T. Uchida, N. Kitamura, and H. Masuhara, Appl. Phys. Lett. 63, 1288 (1993).

${ }^{10}$ N. Kramer, H. Birk, J. Jorritsma, and C. Schonenberger, Appl. Phys. Lett. 66, 1325 (1995)
${ }^{11}$ S. C. Minne, Ph. Flueckiger, H. T. Soh, and C. F. Quate, J. Vac. Sci. Technol. B 13, 1380 (1995).

${ }^{12}$ A. Majumdar, P. I. Oden, J. P. Carrejo, L. A. Nagahara, J. J. Graham, and J. Alexander, Appl. Phys. Lett. 61, 2293 (1992).

${ }^{13}$ H. Sugimura and N. Nakagiri, Jpn. J. Appl. Phys. 34, 698 (1995).

${ }^{14}$ S. C. Minne, H. T. Soh, Ph. Flueckiger, and C. F. Quate, Appl. Phys. Lett. 6, 703 (1995).

${ }^{15}$ P. M. Campbell, E. S. Snow, and J. P. McMarr, Appl. Phys. Lett. 66, 1388 (1995).

${ }^{16}$ K. Matsumoto, M. Ishii, K. Segawa, Y. Oka, B. J. Vartanian, and J. S. Harris, Extended Abstracts of 1994 International Conference of Solid State Device and Materials, Yokohama, Japan, 1994 (unpublished), p. 46.

${ }^{17}$ E. S. Snow and P. M. Campbell, Science 270, 1639 (1995).

${ }^{18}$ S. R. Manalis, S. C. Minne, and C. F. Quate, Appl. Phys. Lett. 68, 871 (1996).

${ }^{19}$ S. R. Manalis, S. C. Minne, A. Atalar, and C. F. Quate, Rev. Sci. Instrum. (to be published).

${ }^{20}$ S. C. Minne, S. R. Manalis, and C. F. Quate, Appl. Phys. Lett. 67, 3918 (1995).

${ }^{21}$ M. Tortonese, Yamada, R. C. Barrett, and C. F. Quate, Proceedings of Transducers '91, IEEE Publ. No. 91 CH2817-5 (IEEE, New York, 1991), p. 448 .

${ }^{22} 10^{20} \mathrm{~cm}^{-3}$ under the $\mathrm{ZnO}, 5 \times 10^{18} \mathrm{~cm}^{-3}$ in the piezoresistor.

${ }^{23}$ Y. Kanada, IEEE Trans. Electron. Devices ED-29, 64 (1982).

${ }^{24}$ S. C. Minne, S. R. Manalis, A. Atalar, and C. F. Quate, Appl. Phys. Lett. 68, 1427 (1996). 